\section{At the centre of things}

\author{
Anthony W. Thomas
}

Models of the Nucleon: From Quarks to Soliton. By Rajat K. Bhaduri. AddisonWesley: 1988. Pp. 360. \$38.95, £34.95.

IT IS no minor task that the author attempts here, namely to present a textbook summary of the various models of nucleon structure, including their experimental basis, for the nuclear physicist. Given the intended audience, quantum field theory is not used; and for the same reason there is an introductory account of gauge theories (including the WeinbergSalam model and quantum chromodynamics)

Perhaps because of the breadth of the task which Bhaduri has set for himself, the quality of the treatment varies enormously. The discussion of deep-inelastic scattering is rather weak and is effectively disconnected from the rest of the book. On the other hand, the non-relativistic quark model and the MIT bag model are well treated. There is a digression into the difference between Dirac and Majorana neutrinos, which seems a little out of place. In the thorough discussion of chiral symmetry, the $\sigma$-model (which is a beautiful textbook example) is endowed with rather too much physical significance. The large- $\mathrm{N}$ expansion is also given too much prominence for my taste, and Witten's conclusion that the nucleon might be a soliton built from weakly interacting meson fields is uncritically repeated.

Some of the nucleon models, particularly the Skyrme model and the ideas of topological solitons, are very clearly explained. This alone will make the book valuable for graduate students working in strong-interaction physics. But one cannot help feeling, as Bhaduri himself suggests, that "it is too early to write a text on these rapidly developing areas". The author has an understandable wish to draw conclusions before it is strictly possible to do so; for example, the point is never made that the main assumption underlying all the topological soliton models is that the effective scalar field confining the quarks is the chiral partner of the pion. This may or may not be true in nature, but to present such models as the apex towards which the whole book is directed is, in my view, misguided.

Nonetheless, this is an impressive piece of work. If read with appropriate caution, it will be prove to be a useful reference volume.

Anthony W. Thomas is a Professor in the Department of Physics and Mathematical Physics, University of Adelaide, PO Box 498, Adelaide, South Australia 5001, Australia.

\title{
Internalizing
}

\section{Pere Alberch}

Morphogenesis and Evolution. By Keith Stewart Thomson. Oxford University Press: 1988. Pp.154. £24, \$29.95.

UNTIL the early 1970 s evolutionary theory was dominated by neo-darwinism, central to which is the idea of adaptation. Organisms evolve by adapting to changes in selective pressures, while at the same time morphological novelties are introduced by genetic mutation. The darwinian view stressed the deterministic properties of natural selection, taking little notice of the problem of how new variation is introduced into a population; that is, how much intrinsic order exists before the action of natural selection.

Data from palaeontology and biochemical systematics now suggest that genetic and developmental regulation may be important sources of novelties in evolution. In consequence, the integration of development and evolution is widely perceived as one of the most exciting and potentially productive 'new' areas of evolutionary biology. As Thomson points out in the book under review, "if we are to progress in evolutionary biology beyond the study only of the contingent, and of unique empirical events, we will need a general theory, and part of that theory must derive from theories of the developmental processes that drive the introduction of variation" (p.69).

The basic idea is that developmental systems are endowed with intrinsic regulatory and pattern-generating properties. The genetic, biochemical, cellular and tissue interactions that characterize developmental systems constrain the realm of possible morphologies, in the sense that even if the source of variation - genetic mutation - is random, its phenotypic expression is not. Certain morphological transformations are more likely to occur than others.

Two corollaries follow. First, the order that we observe in nature results from the interplay of two deterministic factors: internally generated developmental constraints and externally imposed natural selection. Second, if we were to have a good enough knowledge of a specific developmental system we could predict which kind of evolutionary transformation is more likely to occur. Together, constraints on the introduction of phenotypic variation, the limits of selection and predictability in evolutionary processes prescribe a qualitatively new research programme in evolutionary biology, one that concentrates more on the internal organization of the system and less on adaptation.

In recent research on development and evolution, the emphasis has been on mechanisms of morphogenesis, gene regulation and pattern formation rather than on the more traditional approaches based on comparative embryology. Thomson's book emphasizes mechanism over pattern. As the author himself says in the introduction, "the book has nothing to do with the old subject of recapitulation". Instead he concentrates on the role that morphogenetic mechanisms play in evolutionary processes.

Some books in science are useful because they provide a comprehensive review of a specific area, bringing together material from different sources into a unified and clearer context. In others and such books are to be valued even more highly - the author advocates a particular, and novel, thesis. Leo Buss's The Evolution of Individuality (Princeton University Press, 1988) is a recent example:
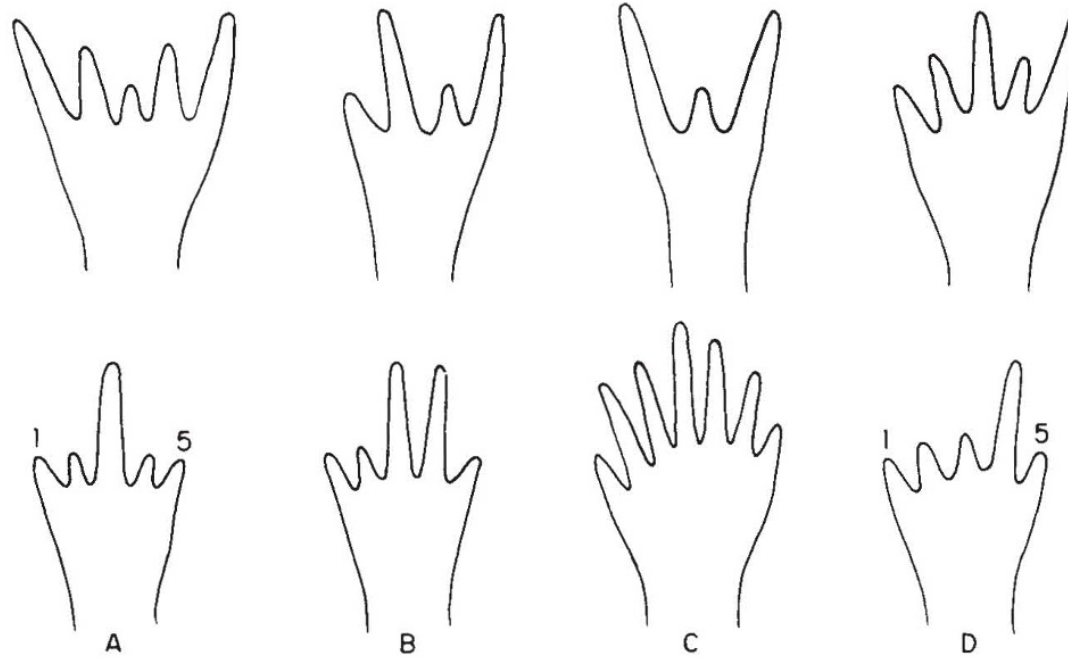

The strong, single axis of orientation in tetrapod limbs means that medial digits persist in evolution, at the expense of lateral ones. The top row shows "forbidden" morphologies: the bottom, "allowed" morphologies. 